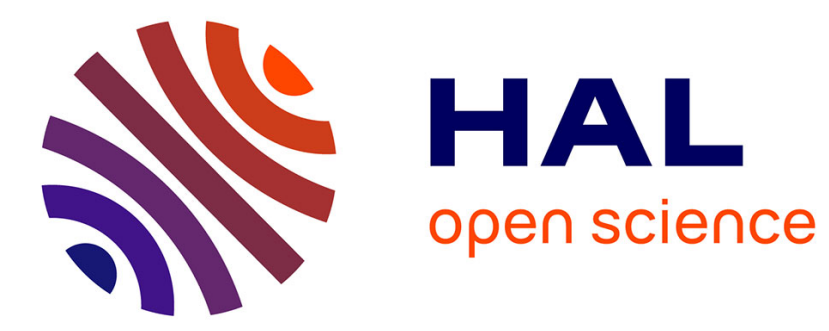

\title{
Stiffness Oriented Tension Distribution Algorithm for Cable-Driven Parallel Robots
}

Etienne Picard, Stéphane Caro, Franck Plestan, Fabien Claveau

\section{To cite this version:}

Etienne Picard, Stéphane Caro, Franck Plestan, Fabien Claveau. Stiffness Oriented Tension Distribution Algorithm for Cable-Driven Parallel Robots. In: Lenarcic J., Siciliano B. (eds) Advances in Robot Kinematics 2020. ARK 2020. Springer Proceedings in Advanced Robotics, 15, pp.209-217, 2020, 978-3-030-50974-3. 10.1007/978-3-030-50975-0_26 . hal-02515911

\section{HAL Id: hal-02515911 \\ https://hal.science/hal-02515911}

Submitted on 23 Mar 2020

HAL is a multi-disciplinary open access archive for the deposit and dissemination of scientific research documents, whether they are published or not. The documents may come from teaching and research institutions in France or abroad, or from public or private research centers.
L'archive ouverte pluridisciplinaire HAL, est destinée au dépôt et à la diffusion de documents scientifiques de niveau recherche, publiés ou non, émanant des établissements d'enseignement et de recherche français ou étrangers, des laboratoires publics ou privés. 


\title{
Stiffness Oriented Tension Distribution Algorithm for Cable-Driven Parallel Robots
}

\author{
Etienne Picard, Stéphane Caro, Franck Plestan and Fabien Claveau
}

\begin{abstract}
A novel criterion is introduced in this paper to determine the set of cable tensions for Cable-Driven Parallel Robots (CDPRs) with the aim of maximizing the robot stiffness along a specific direction. Based on the feasible polygon of the CDPR and its stiffness matrix, an algorithm selects the set of admissible cable tensions leading to the smallest moving-platform displacement, the moving-platform being subject to an external wrench. The proposed tension distribution is implemented in a control scheme and experimented on a fully-constrained CDPR for a window cleaning application.
\end{abstract}

Key words: Cable-driven parallel robot, Tension Distribution Algorithm, Stiffness, Control, Experiments

\section{Introduction}

Cable-Driven Parallel Robots (CDPRs) are a particular class of parallel robots whose moving-platform (MP) is connected to a fixed base frame by cables, as illustrated in Fig. 1. Both the orientation and the position of the MP are controlled by

Etienne Picard

IRT Jules Verne, Bouguenais, 44340, France

e-mail:etienne.picardeirt-jules-verne.fr

Stéphane Caro

Centre National de la Recherche Scientifique (CNRS), Nantes, 44321 France

e-mail: stephane.carodls2n.fr

Franck Plestan

École Centrale de Nantes - LS2N, UMR CNRS 6004, Nantes, 44321 France

e-mail: franck.plestandec-nantes.fr

Fabien Claveau

IMT-Atlantique - LS2N, UMR CNRS 6004, Nantes, 44300 France

e-mail: fabien.claveaudimt-atlantique.fr 
cables coiled on motorized winches. Passive pulleys may guide the cables from the winches to the cable exit points. CDPRs have several advantages such as a relatively low mass of moving parts, high dynamics and a potential large workspace. As a consequence, they can be used in several applications such as heavy load handling [1], painting and sandblasting of large structures [8], fast pick-and-place operations [11], haptic devices [6], support structures for giant telescopes [18], and search and rescue deployable platforms [14].

However, the use of cables introduces specific control challenges. In particular, the position control of fully-constrained CDPRs, whose cables coming from both the top and the bottom of the frame, requires more attention than purely suspended ones. Small errors in the computation of the necessary cable lengths to achieve a desired MP pose can lead to slack cables or high tensions in opposing cables. A tension distribution algorithm (TDA) can then be implemented to balance a wrench between all cables while maintaining the static or dynamic equilibrium of the MP, and to enforce boundaries on the cable tensions. The most commonly used TDAs for calculating the force distribution among the cables of classical CDPRs are the following: (i) Gradient-based optimization approach [17]; (ii) Quadratic programming [5]; (iii) Linear programming Methods [4]; (iv) Minimizing norm solution with Dykstra method [9]; (v) Centroid/Barycentric approach [13]. A comparison between existing TDAs is given in [16]. It is noteworthy that the cable tension distribution is influenced by the cable elasticity and the existing TDAs do not consider the CDPR stiffness. Accordingly, the main contribution of this paper lies in the cable tension distribution based on a CDPR stiffness performance index, namely, a novel Stiffness Oriented Tension Distribution Algorithm (SOTDA).

A CDPR prototype was assembled in the framework of the IRT Jules Verne ROCKET project for a window cleaning application, denoted as ROWC (ROCKET Window Cleaning) and shown in Fig. 1. The moving-platform is equipped with a pressure washer hose. In operation, pressurized water is projected onto the surface to clean, which creates a wrench onto the MP in the opposite direction of the water flow. Due to cable elasticity, this external wrench induces a small displacement of the MP along the normal to the plane of MP motion.

In the sequel, the ROWC prototype and its modeling are introduced in Sec. 2. Then, the SOTDA is described in Sec. 3 . Experimental results on ROWC obtained with a control scheme integrating the SOTDA are presented in Sec. 4

\section{Prototype and modeling}

Figure 1 presents the prototype projecting water onto a facade. This CDPR uses a fully-constrained cable configuration with eight cables and six degrees of freedom. A video of the window cleaning application is available 1 . This prototype is actuated by $B \& R$ Automation synchronous motors of nominal speed and nominal torque

1 ROCKET window cleaning application video (link) 
equal to $2100 \mathrm{rpm}$ and $2.6 \mathrm{Nm}$, respectively. The motors are coupled to planetary gearboxes with a reduction ratio of 5 , and its winches have a diameter of $90 \mathrm{~mm}$. A specific platform was designed for the window cleaning application, equipped with a water nozzle located at the extremity of a deployable mechanism. Due to its cable configuration, the prototype has an almost planar workspace and presents the lowest stiffness along the $y_{b}$ axis, aligned with the direction of force due to water pressure. As a consequence, the SOTDA aims to select the set of cable tensions that maximizes the stiffness of the MP along the $y_{b}$ axis.

Figure 1 depicts the main geometric parameters of the CDPR at hand. Cable exit points are denoted as $A_{i}$, while cable anchor points are denoted as $B_{i}$, with $i \in\{1, \ldots, m\}, m$ being the number of cables attached to the MP, $m=8$ for ROWC. $\mathscr{F}_{b}$ is the robot base frame, and $\mathscr{F}_{p}$ is the MP frame. Vector ${ }^{b} \mathbf{a}_{i}$ points from $O$ to $A_{i}$ and is expressed in frame $\mathscr{F}_{b}$. Vector ${ }^{p} \mathbf{b}_{i}$ point from $P$ to $B_{i}$ and is expressed in frame $\mathscr{F}_{p}$. Vector ${ }^{b} \mathbf{u}_{i}$ is the $i^{\text {th }}$ cable unit vector pointing from $B_{i}$ to $A_{i}$. Vector ${ }^{b} \mathbf{p}$ is the position vector of point $P$, the MP geometric center, expressed in $\mathscr{F}_{b}$. As the pulleys of the ROWC prototype are very small compared to the cable lengths, the following model is established from a geometric modeling neglecting the pulleys.
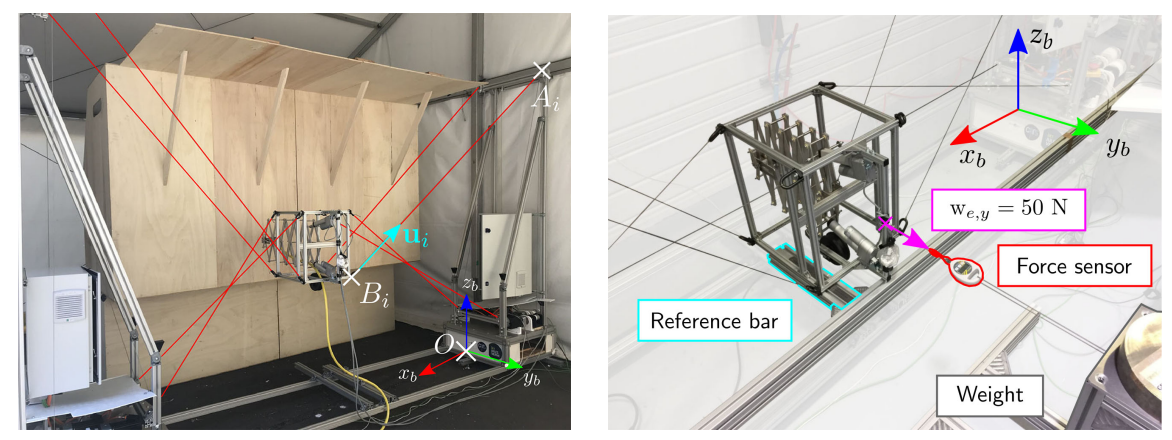

Fig. 1: (Left.) ROWC prototype projecting pressurized water onto a boat model (Right.) Experimental setup with lateral wrench for the static case.

The static equilibrium of the MP is given by

$$
\mathbf{W t}+\mathbf{w}_{e}+\mathbf{w}_{g}=0
$$

where $\mathbf{t}$ is the cable tension vector, $\mathbf{w}_{g}$ the wrench applied to the MP due to gravity, $\mathbf{w}_{e}$ an external wrench expressed in frame $\mathscr{F}_{b}$, and $\mathbf{W}$ the wrench matrix of the robot, whose expression is given by:

$$
\mathbf{W}=\left[\begin{array}{ccccc}
{ }^{b} \mathbf{u}_{1} & \ldots & { }^{b} \mathbf{u}_{i} & \ldots & { }^{b} \mathbf{u}_{m} \\
{ }^{b} \mathbf{b}_{1} \times{ }^{b} \mathbf{u}_{1} & \ldots & { }^{b} \mathbf{b}_{i} \times{ }^{b} \mathbf{u}_{i} & \ldots & { }^{b} \mathbf{b}_{m} \times{ }^{b} \mathbf{u}_{m}
\end{array}\right]
$$

The stiffness matrix $\mathbf{K}$ of ROWC is expressed based on a linear cable elasticity model [2]. From [3] and [7], $\mathbf{K}$ is defined as: 


$$
\mathbf{K}=\frac{\delta \mathbf{w}_{\mathbf{e}}}{\delta \mathbf{X}}=\mathbf{K}_{p}+\mathbf{K}_{a} \quad \text { with } \quad \delta \mathbf{X}=[\delta \mathbf{p} \delta \phi]^{\mathrm{T}}
$$

where a small change in the external wrench $\delta \mathbf{w}_{e}$ results in a small displacement screw $\delta \mathbf{X}$ of the MP. $\delta \mathbf{p}$ and $\delta \phi$ are respectively the translational and rotational components of the displacement. $\mathbf{K}_{p}$ and $\mathbf{K}_{a}$ are named passive and active stiffness matrices, respectively, and take the form:

$$
\begin{aligned}
& \mathbf{K}_{p}=\sum_{i=1}^{m} k_{i}\left[\begin{array}{cc}
{ }^{b} \mathbf{u}_{i}{ }^{b} \mathbf{u}_{i}^{\mathrm{T}} & \left.{ }^{b} \mathbf{u}_{i}{ }^{b} \mathbf{b}_{i}{ }^{\mathrm{T}}{ }_{i} \hat{\mathbf{u}}_{i}{ }^{\mathrm{b}} \mathbf{u}_{i}^{\mathrm{T}}{ }^{\mathrm{T}} \hat{\mathbf{b}}_{i}{ }^{b} \mathbf{u}_{i}{ }^{b} \mathbf{u}_{i}^{\mathrm{T}} \hat{\mathbf{b}}_{i}^{\mathrm{T}}\right]
\end{array}\right] \\
& \mathbf{K}_{a}=-\sum_{i=1}^{m} t_{i}\left[\begin{array}{cc}
-\frac{1}{l_{i}}\left(\mathbf{I}_{3}-{ }^{b} \mathbf{u}_{i}{ }^{b} \mathbf{u}_{i}^{\mathrm{T}}\right) & \frac{1}{l_{i}}\left(\mathbf{I}_{3}-{ }^{b} \mathbf{u}_{i}{ }^{b} \mathbf{u}_{i}{ }^{\mathrm{T}}\right){ }^{b} \hat{\mathbf{b}}_{i} \\
-\frac{1}{l_{i}}{ }^{b} \hat{\mathbf{b}}_{i}\left(\mathbf{I}_{3}-{ }^{b}{ }_{\mathbf{u}_{i}}{ }^{b} \mathbf{u}_{i}{ }^{\mathrm{T}}\right) & {\left[\begin{array}{c}
\left.{ }^{b} \hat{\mathbf{u}}_{i}+\frac{{ }^{b} \hat{\mathbf{b}}_{i}}{l_{i}}\left(\mathbf{I}_{3}-{ }^{b} \mathbf{u}_{i}{ }^{b} \mathbf{u}_{i}{ }^{\mathrm{T}}\right)\right]
\end{array}\right]{ }^{b} \hat{\mathbf{b}}_{i}}
\end{array}\right]
\end{aligned}
$$

with $k_{i}$ the $i^{t h}$ cable stiffness and $t_{i}$ its tension. $\mathbf{K}_{p}$ is a function of the cable elasticity, whereas $\mathbf{K}_{a}$ depends on cable tensions.

\section{Stiffness Oriented Tension Distribution Algorithm}

A solution to tension distribution is to find a set of cable tensions ensuring the static equilibrium of the MP. For over-constrained CDPRs with an actuation redundancy degree equal to two, like ROWC, the set of admissible solutions is characterized by the the polygon of feasible tensions [10]. Let $t_{\min }$ and $t_{\max }$ be the lower and upper cable tension limits. A solution to the static equilibrium (Eq. (1)) can be written as:

$$
\mathbf{t}=\mathbf{W}^{\dagger} \mathbf{f}+\mathbf{N} \lambda \quad \text { with } \quad \lambda=\left[\begin{array}{ll}
\lambda_{1} & \lambda_{2}
\end{array}\right]^{\mathrm{T}}
$$

where $\mathbf{N}=\operatorname{null}(\mathbf{W})$ is the null space of the wrench matrix $\mathbf{W}$, and $\lambda$ is an arbitrary vector of coordinates $\lambda_{1}$ and $\lambda_{2}$. Let $\Sigma$ be the 2D space of solutions $\left[\begin{array}{ll}\lambda_{1} & \lambda_{2}\end{array}\right]^{\mathrm{T}}$ obtained from Eq. $(5)$, and $\Omega$ the hypercube of feasible cable tensions $\Omega=\left\{\mathbf{t} \mid 0 \leqslant t_{\text {min }} \leqslant t_{i} \leqslant t_{\text {max }}, i=1, \ldots, m\right\}$. Their intersection $\Lambda=\Sigma \cap \Omega$ is a $2 \mathrm{D}$ convex polytope of feasible cable tensions sets, obtained from the affine map $\mathscr{A}=\left(\mathbf{N}, \mathbf{t}_{0}\right)$ :

$$
\mathscr{A}^{-1}(\Lambda)=\left\{\lambda \in \mathbb{R}^{2} \mid \mathbf{t}_{\text {min }} \leqslant \mathbf{t}_{0}+\mathbf{N} \lambda \leqslant \mathbf{t}_{\text {max }}\right\}
$$

with $\mathbf{t}_{0}=\mathbf{W}^{\dagger} \mathbf{f} . \mathscr{A}^{-1}(\Lambda)$ is also a $2 \mathrm{D}$ convex polytope, named feasible polygon.

The proposed TDA aims to minimize the moving-platform translational displacement $\delta \mathbf{p}$ due to an external wrench $\delta \mathbf{w}_{e}$ exerted onto the MP, along a given direction. From Eq. (3), if the goal is to maximize the MP stiffness along axis $y_{b}$, the SOTDA will have to minimize the following objective function: 


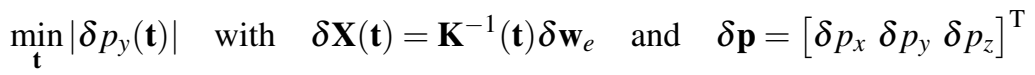

where the cable tension vector $\mathbf{t}$ is a function of $\lambda_{1}$ and $\lambda_{2}$, and $\delta \mathbf{w}_{e}$ is a small wrench in the same direction of the external wrench $\mathbf{w}_{e}$. The corresponding optimization problem can be solved by different methods, such as a nonlinear least square method.

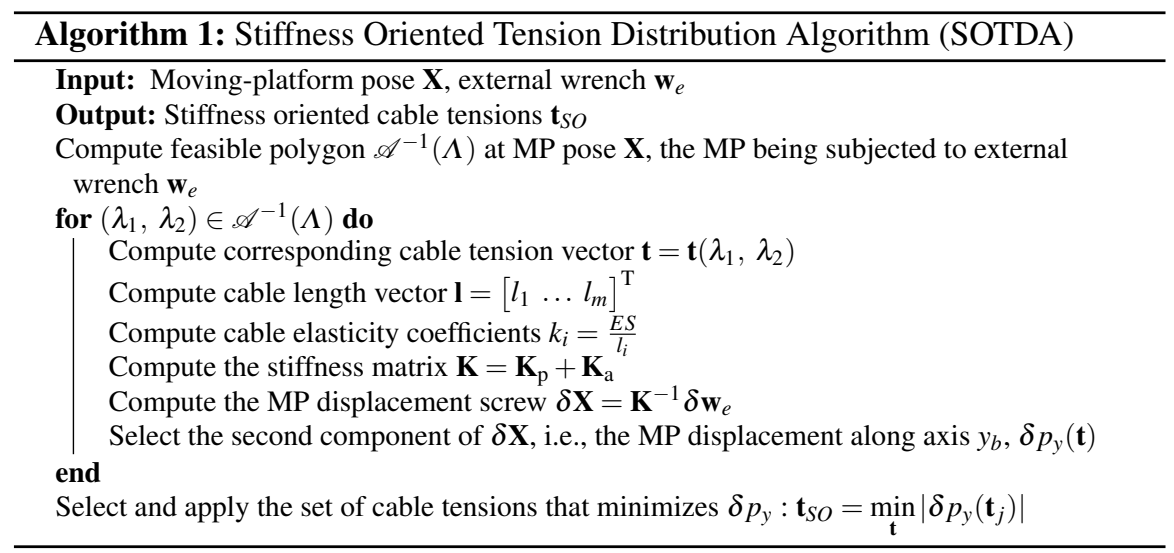

Algorithm 1 describes the proposed SOTDA, $\mathbf{t}_{S O}$ being the resulting cable tension vector. For a real-time implementation, two assumptions are made to reduce the number of sets $\left(\lambda_{1}, \lambda_{2}\right)$ to be evaluated and the computing time: $(i)$ the robot has the highest stiffness when at least one cable tension reaches the upper bound $t_{\max }$; (ii) a second cable should either reach the lower cable tension limit $t_{\min }$ or the upper cable tension limit $t_{\max }$. Under these assumptions, the optimal solution should then be located at the intersection of two vertices, that is at a corner of the feasible polygon. For an 8-cable CDPR with an actuation redundancy equal to two, these assumptions lead to a maximum of 16 sets $\left(\lambda_{1}, \lambda_{2}\right)$ to be evaluated. It is noteworthy that even if the optimal solution is not amongst the set of solutions, the algorithm will consider the best of the computed ones.

In the sequel, an Intermediate Solution (IS) is introduced by selecting a new point between the barycenter of the feasible polygon and the stiffness oriented solution, based on the value of a coefficient $v$ bounded between 0 and 1 . The implementation of $v$ defines a security margin with respect to the edges of the feasible polygon as shown in Fig. 2 


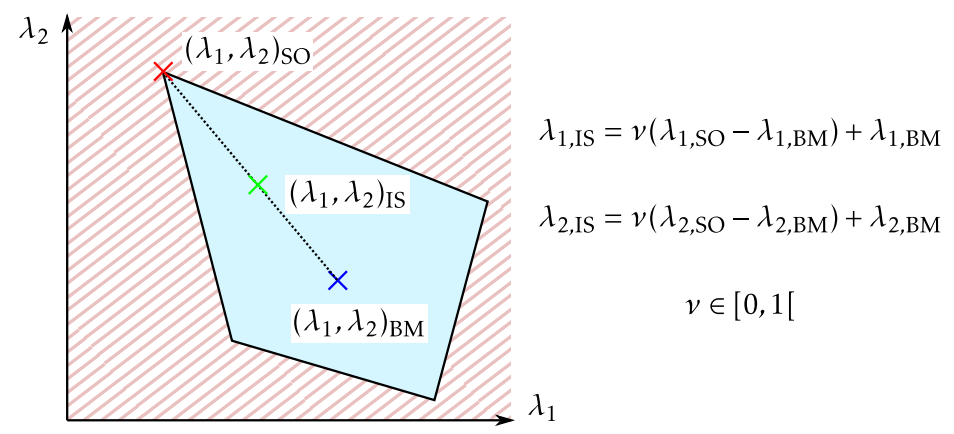

Fig. 2: Intermediate point (IS) inside the feasible polygon

\section{Experimental results}

Similarly to the implementation of classical Tension Distribution Algorithms (TDAs) [12 15], the SOTDA is located in the control scheme after the sum of the control signal $\tau_{c}$ and feedforward term $\tau_{d a}$, as illustrated in Fig. 3 A proportional-derivative (PD) controller is chosen for its asymptotic stability, tuned according to the ZieglerNichols method.

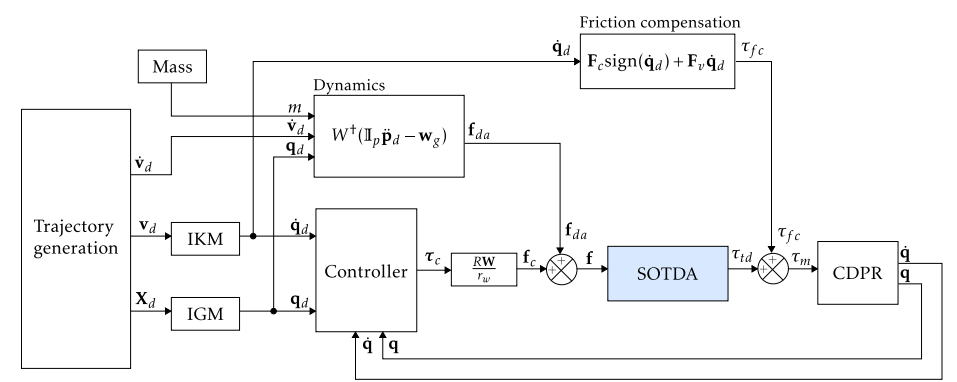

Fig. 3: Control scheme with SOTDA on controller and feedforward block outputs.

The force magnitude due to water jet in the actual cleaning application is about $12.5 \mathrm{~N}$ along the $y_{b}$ axis. In the following, forces of $30 \mathrm{~N}$ and $50 \mathrm{~N}$ are applied on the MP for better observation of the MP behavior. First, the SOTDA is experimented in a static pose $\mathbf{X}_{0}=\left[\begin{array}{llllll}1.5 & 0.350 & 0.590 & 0 & 0 & 0\end{array}\right]^{\mathrm{T}}$ to validate the stiffness gain under

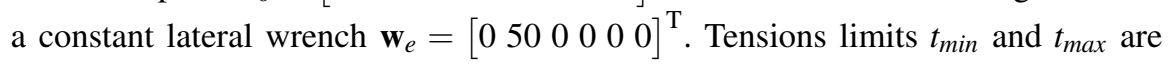
respectively set to $10 \mathrm{~N}$ and $200 \mathrm{~N}$. The Young modulus is $E=80 \mathrm{GPa}$ and the cross-section area of the cables is $S=0.75 \mathrm{~mm}^{2}$. Figure 4 presents the estimation in simulation of the MP displacement $\delta p_{y}$ within the entire feasible polygon and the experimental results obtained on the real prototype. 
From Eq. (7), the estimation of the MP displacement requires the definition of a small external wrench $\delta \mathbf{w}_{e}$ along the direction in which the stiffness must be maximized. Here, $\delta \mathbf{w}_{e}$ amounts to the total external wrench due to water pressure. Using MATLAB meshgrid function, the feasible polygon is discretized and the MP displacement along the $y$ axis, $\delta p_{y}$, is computed for all points of the grid. The vertical colorbar presents the value of $\delta p_{y}$ in mm within the feasible polygon.
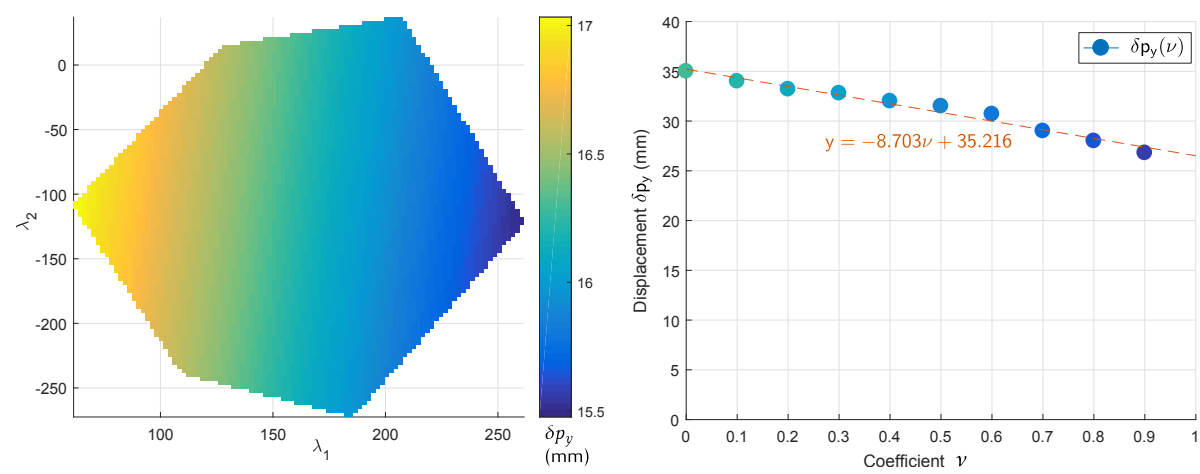

Fig. 4: (Left) Estimated MP displacement $\delta p_{y}(\mathrm{~mm})$ within the feasible polygon (Right) Measured MP displacement $\delta p_{y}(\mathrm{~mm})$ as a function of coefficient $v$.

From the simulation results, the computed displacements show a difference of $1.5 \mathrm{~mm}$ between the lowest and highest values. It is noteworthy that the influence of the solution selection on the MP displacement is small compared to the minimal total error of $15.5 \mathrm{~mm}$. It means that the passive part $\mathbf{K}_{p}$ of the stiffness matrix $\mathbf{K}$ might far outweighs its active part $\mathbf{K}_{a}$.

The experimental process in the static case is as follows:

1. The MP is positioned next to a fixed reference bar, as shown in Fig.1;

2. Using a steel scribe, the resting position of the MP is marked on the reference bar when no external wrench is applied;

3. For each value of $v=\{0,0.1,0.2, \ldots, 0.9\}$, a lateral wrench of $30 \mathrm{~N}$ is applied onto the MP along $y_{b}$-axis, using a Kern HDB $10 \mathrm{~K} 10 \mathrm{~N}$ force sensor. Then, the new steady position of the MP is marked on the bar.

The resulting MP displacements as a function of coefficient $v$ are plotted in Fig. 4 It is apparent that the higher $v$, the smaller the MP displacement. The SOTDA leads a reduction of up to $24.8 \%$ of the displacement due to the lateral force compared to the solution obtained by selecting the feasible polygon barycenter to tune the cable tensions. With values bounded between 26 and $36 \mathrm{~mm}$, the measured MP displacements are larger than the estimated ones. This difference is due to the modeling simplifications while neglecting the pulleys geometry and the choice of a linear elastic cable model. The simulation results also only consider the elasticity of the 
cable, while neglecting the compliance of the controller and the elasticity of the frame. Therefore, the real MP displacements are larger than the simulated ones.

In a second experiment, the MP follows the linear path shown in Fig. 5. An external force equal to $30 \mathrm{~N}$ is exerted onto the MP along the trajectory. The upper cable tension limit $t_{\max }$ is set to $150 \mathrm{~N}$ and the trajectory is performed for $v=0$ and $v=0.9$, respectively. The cable tension profiles obtained with the SOTDA for $v=0$ and $v=0.9$, respectively, are plotted in Fig. 5 .
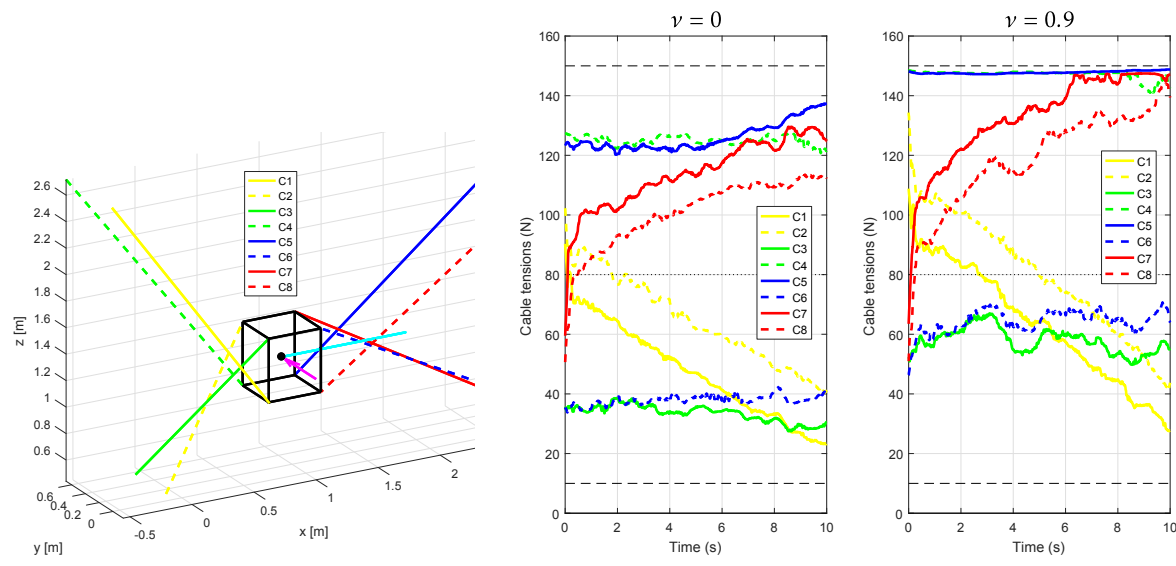

Fig. 5: (Left) Test path (cyan) (Right) Cable tensions profiles, i.e., SOTDA output, for $v=0$ and $v=0.9$, resp.

On the one hand, two cable tensions are close to $t_{\max }$ at all time when $v=0.9$. Cables 4 and 5 are the most tensed ones along the trajectory since they oppose the external wrench and support the MP weight. As a result, their tensions are close to $t_{\max }$, while maintaining the MP static equilibrium. On the other hand, cable tensions are around the average admissible cable tension of $80 \mathrm{~N}$ for $v=0$. Besides, it is should be noted that cable tensions remain continuous with the SOTDA. The value of $v$ can then be selected to find a trade-off between the stiffness oriented solution and the one obtained with the barycenter approach.

\section{Conclusion}

In this paper, a stiffness oriented tension distribution algorithm (SOTDA) was proposed to select the set of cable tensions of a CDPR minimizing the displacement of its moving-platform, the latter being subject to a lateral force along a given direction. The algorithm was studied in simulation and tested experimentally on a fully-constrained CDPR. The experimental results show that the moving-platform displacement can be reduced by up to $24 \%$ with a good selection of cable tensions. 
It is expected that a higher upper tension limit could further improve the benefits of the SOTDA. The introduction of the stiffness coefficient $v$ for the selection of an intermediate point allows for the implementation of the SOTDA while maintaining a safety margin with respect to the maximum feasible tensions. Future work includes a sensitivity analysis of the SOTDA parameters such as tension limits and cable configuration, a finer identification of the CDPR elastic properties and experiments with a variable pressure water jet.

Acknowledgements The authors wish to associate the industrial and academic partners of IRT Jules Verne in the framework of the ROCKET project, namely Chantiers de l'Atlantique, Clemessy, B\&R Automation, Clarté and CNRS.

\section{References}

1. Albus, J., Bostelman, R., Dagalakis, N.: The NIST SPIDER, A robot crane. Journal of Research of the National Institute of Standards and Technology volume 97(3), 373 (1992)

2. Baklouti, S., Courteille, E., Caro, S., Dkhil, M.: Dynamic and Oscillatory Motions of CableDriven Parallel Robots Based on a Nonlinear Cable Tension Model. Journal of Mechanisms and Robotics 9(6), 061,014 (2017)

3. Behzadipour, S.: Stiffness of Cable-based Parallel Manipulators With Application to Stability Analysis. Journal of mechanical design (2006)

4. Borgstrom, P.H., Jordan, B.L., Sukhatme, G.S., Batalin, M.A., Kaiser, W.J.: Rapid computation of optimally safe tension distributions for parallel cable-driven robots. IEEE Transactions on Robotics 25(6), 1271-1281 (2009)

5. Bruckmann, T., Pott, A., Hiller, M.: Calculating force distributions for redundantly actuated tendon-based stewart platforms. In: J. Lennarčič, B. Roth (eds.) Advances in Robot Kinematics, pp. 403-412. Springer Netherlands, Dordrecht (2006)

6. Fortin-Coté, A., Cardou, P., Gosselin, C.: An admittance control scheme for haptic interfaces based on cable-driven parallel mechanisms. In: Proceedings of the 2014 IEEE International Conference on Robotics and Automation (ICRA), pp. 819-925. Hong Kong (2014)

7. Gagliardini, L., Caro, S., Gouttefarde, M., Girin, A.: Discrete reconfiguration planning for Cable-Driven Parallel Robots. Mechanism and Machine Theory 100, 313-337 (2016)

8. Gagliardini, L., Gouttefarde, M., Caro, S.: Determination of a Dynamic Feasible Workspace for Cable-Driven Parallel Robots. Advances in Robot Kinematics 2016 volume 4, 361-370 (2018)

9. Hassan, M., Khajepour, A.: Minimum-norm solution for the actuator forces in cable-based parallel manipulators based on convex optimization. In: Proceedings 2007 IEEE International Conference on Robotics and Automation, pp. 1498-1503 (2007)

10. Hassan, M., Khajepour, A.: Analysis of bounded cable tensions in cable-actuated parallel manipulators. IEEE Transactions on Robotics (2011)

11. Kawamura, S., Kino, H., Won, C., Kamawura, S., Kino, H., Won, C.: High-speed manipulation by using parallel wire-driven robots. Robotica 18(1), 13-21 (2000)

12. Lamaury, J.: Contribution a la commande des robots paralleles a cables a redondance d'actionnement (2013)

13. Lamaury, J., Gouttefarde, M.: A Tension Distribution Method with Improved Computational Efficiency, pp. 71-85. Springer Berlin Heidelberg, Berlin, Heidelberg (2013)

14. Merlet, J.P., Daney, D.: A portable, modular parallel wire crane for rescue operations. In: Proceedings of the 2010 IEEE International Conference on Robotics and Automation (ICRA), pp. 2834-2839 (2010) 
15. Picard, , Caro, S., Claveau, F., Plestan, F.: Pulleys and force sensors influence on payload estimation of cable-driven parallel robots. In: 2018 IEEE/RSJ International Conference on Intelligent Robots and Systems (IROS), pp. 1429-1436 (2018)

16. Pott, A.: An improved force distribution algorithm for over-constrained cable-driven parallel robots. In: F. Thomas, A. Perez Gracia (eds.) Computational Kinematics, pp. 139-146. Springer Netherlands, Dordrecht (2014)

17. Verhoeven, R.: Analysis of the workspace of tendon based Stewart platforms. Phd thesis, verlag nicht ermittelbar. (2004)

18. Yao, R., Tang, X., Wang, J., Huang, P.: Dimensional optimization design of the four-cabledriven parallel manipulator in fast. IEEE/ASME Transactions on Mechatronics 15(6), 932941 (2010) 